Supplementary File S5: Taxonomy and Core Microbiome Analysis

Bacterial Taxonomy

Table S5a: Bacterial Taxonomic classification at Phylum level

\begin{tabular}{|r|r|r|r|}
\hline Taxa & Abundance & Percentage \\
\hline 1 p_Proteobacteria & 212887 & 97.67 \\
\hline 3 p_Actinobacteria & 2455 & 1.13 \\
\hline 2 p__Bacteroidetes & 1816 & 0.83 \\
\hline 4 p_Firmicutes & 719 & 0.33 \\
\hline 5 Unclassified & 83 & 0.04 \\
\hline
\end{tabular}

Table S5b: Bacterial Taxonomic Classification at Class level

\begin{tabular}{|r|l|r|r|}
\hline & Taxa & Abundance & Percentage \\
\hline 1 & c_Gammaproteobacteria & 154928 & 71.04 \\
\hline 3 & c_Alphaproteobacteria & 57835 & 26.52 \\
\hline 4 & c_Actinobacteria & 2472 & 1.13 \\
\hline 2 & c_Bacteroidia & 1832 & 0.84 \\
\hline 6 & c_Bacilli & 606 & 0.28 \\
\hline 8 & Not_Assigned & 294 & 0.13 \\
\hline 5 & c_Clostridia & 86 & 0.04 \\
\hline 7 & c_Erysipelotrichia & 27 & 0.01 \\
\hline
\end{tabular}

Table S5c: Bacterial Taxonomic Classification at Order level

\begin{tabular}{|r|l|r|r|}
\hline & Taxa & Abundance & Percentage \\
\hline 3 & o__Betaproteobacteriales & 94938 & 43.53 \\
\hline 10 & o__Pseudomonadales & 51984 & 23.84 \\
\hline 4 & o_Caulobacterales & 27903 & 12.79 \\
\hline 12 & o__Sphingomonadales & 20246 & 9.28 \\
\hline 11 & o__Rhizobiales & 8664 & 3.97 \\
\hline 8 & o__Oceanospirillales & 2958 & 1.36 \\
\hline 13 & o_Xanthomonadales & 2396 & 1.1 \\
\hline 14 & Others & 1785 & 0.82 \\
\hline 6 & o_Cytophagales & 1561 & 0.72 \\
\hline 2 & o_Alteromonadales & 1414 & 0.65 \\
\hline 7 & o_Micrococcales & 1360 & 0.62 \\
\hline 1 & Not_Assigned & 1100 & 0.5 \\
\hline 5 & o_CCorynebacteriales & 929 & 0.43 \\
\hline 9 & o__Parvibaculales & 842 & 0.39 \\
\hline
\end{tabular}


Table S5d: Bacterial Taxonomic Classification at Family Level

\begin{tabular}{|r|l|r|r|}
\hline & Taxa & Abundance & Percentage \\
\hline 1 & f__Burkholderiaceae & 94938 & 43.53 \\
\hline 2 & f__Pseudomonadaceae & 48578 & 22.28 \\
\hline 3 & f__Caulobacteraceae & 27903 & 12.79 \\
\hline 4 & f__Sphingomonadaceae & 20246 & 9.28 \\
\hline 5 & f__Rhizobiaceae & 6572 & 3.01 \\
\hline 6 & f__Moraxellaceae & 3406 & 1.56 \\
\hline 7 & f__Halomonadaceae & 2851 & 1.31 \\
\hline 8 & f__Xanthomonadaceae & 2396 & 1.1 \\
\hline 9 & f__Hymenobacteraceae & 1561 & 0.72 \\
\hline 10 & f__Shewanellaceae & 1414 & 0.65 \\
\hline 11 & f__Devosiaceae & 1286 & 0.59 \\
\hline 12 & Not_Assigned & 1100 & 0.5 \\
\hline 13 & f__Corynebacteriaceae & 929 & 0.43 \\
\hline 14 & f__Parvibaculaceae & 842 & 0.39 \\
\hline 15 & f__Microbacteriaceae & 690 & 0.32 \\
\hline 16 & f__Beijerinckiaceae & 658 & 0.3 \\
\hline 17 & f__Micrococcaceae & 484 & 0.22 \\
\hline 18 & f__Solimonadaceae & 432 & 0.2 \\
\hline 19 & f__Staphylococcaceae & 321 & 0.15 \\
\hline 20 & f__Bacillaceae & 285 & 0.13 \\
\hline 21 & f__Flavobacteriaceae & 271 & 0.12 \\
\hline 22 & f__Intrasporangiaceae & 156 & 0.07 \\
\hline 23 & f__Propionibacteriaceae & 153 & 0.07 \\
\hline 24 & f__Xanthobacteraceae & 148 & 0.07 \\
\hline 25 & f__Rhodobacteraceae & 137 & 0.06 \\
\hline 26 & f__Hahellaceae & 82 & 0.04 \\
\hline 27 & f__Peptostreptococcaceae & 49 & 0.02 \\
\hline 28 & f__Azospirillaceae & 43 & 0.02 \\
\hline 29 & f__Clostridiaceae_1 & 37 & 0.02 \\
\hline 30 & f__Dermabacteraceae & 30 & 0.01 \\
\hline 31 & f_Erysipelotrichaceae & 27 & 0.01 \\
\hline 32 & f__SS1_B_06_26 & 25 & 0.01 \\
\hline 33 & f__Nocardioidaceae & 15 & 0.01 \\
\hline 34 & f__Geodermatophilaceae & 15 & 0.01 \\
\hline & & & \\
\hline & & & \\
\hline & & & \\
\hline
\end{tabular}


Table S5e: Bacterial Taxonomic Classification at Genus level

\begin{tabular}{|r|l|r|r|}
\hline & Taxa & Abundance & Percentage \\
\hline 1 & Not_Assigned & 127725 & 58.57 \\
\hline 2 & g__Brevundimonas & 27248 & 12.49 \\
\hline 3 & g__Sphingobium & 7196 & 3.3 \\
\hline 4 & g__Sphingopyxis & 6035 & 2.77 \\
\hline 5 & g__Pseudomonas & 5448 & 2.5 \\
\hline 6 & g__Sphingomonas & 5265 & 2.41 \\
\hline 7 & g__Massilia & 5143 & 2.36 \\
\hline 8 & g__Acidovorax & 4461 & 2.05 \\
\hline 9 & g__Allorhizobium & 3840 & 1.76 \\
\hline 10 & g__Halomonas & 2851 & 1.31 \\
\hline 11 & g__Mesorhizobium & 2492 & 1.14 \\
\hline 12 & g__Novosphingobium & 1750 & 0.8 \\
\hline 13 & g__Pseudoxanthomonas & 1709 & 0.78 \\
\hline 14 & g__Enhydrobacter & 1606 & 0.74 \\
\hline 15 & g__Pontibacter & 1561 & 0.72 \\
\hline 16 & g__Shewanella & 1414 & 0.65 \\
\hline 17 & g__Cavicella & 1404 & 0.64 \\
\hline 18 & g__Devosia & 1286 & 0.59 \\
\hline 19 & g__Achromobacter & 1015 & 0.47 \\
\hline 20 & g__Corynebacterium_1 & 929 & 0.43 \\
\hline 21 & g__Parvibaculum & 842 & 0.39 \\
\hline 22 & g__Delftia & 822 & 0.38 \\
\hline 23 & g__Stenotrophomonas & 687 & 0.32 \\
\hline 24 & g__Rhizobacter & 585 & 0.27 \\
\hline 25 & g__Microvirga & 82 & 0.22 \\
\hline 26 & g__uncultured & 484 & 0.21 \\
\hline 27 & g__Variovorax & 463 & 0.04 \\
\hline 28 & g__Azotobacter & 461 & 0.21 \\
\hline 29 & g__Hydrocarboniphaga & 447 & 0.2 \\
\hline 30 & g__Pseudarthrobacter & 432 & 0.2 \\
\hline 31 & g__Staphylococcus & 339 & 0.16 \\
\hline 32 & g__Bacillus & 285 & 0.15 \\
\hline 33 & g__Salinimicrobium & 271 & 0.13 \\
\hline 34 & g__Methylobacterium & 174 & 0.12 \\
\hline 35 & g__Tetrasphaera & 156 & 0.08 \\
\hline 36 & g__Cutibacterium & 153 & 0.07 \\
\hline 37 & g__Bradyrhizobium & 148 & 0.07 \\
\hline 38 & g__Kocuria & 0.07 \\
\hline 39 & g__Hahella & & 0.04 \\
\hline & & & \\
\hline
\end{tabular}




\begin{tabular}{|r|l|r|r|}
\hline 40 & g__Paracoccus & 80 & 0.04 \\
\hline 41 & g__Ramlibacter & 69 & 0.03 \\
\hline 42 & g__Micrococcus & 50 & 0.02 \\
\hline 43 & g__Romboutsia & 49 & 0.02 \\
\hline 44 & g__Skermanella & 43 & 0.02 \\
\hline 45 & g__Noviherbaspirillum & 40 & 0.02 \\
\hline 46 & g__Clostridium_sensu_stricto_1 & 37 & 0.02 \\
\hline 47 & g__Brachybacterium & 30 & 0.01 \\
\hline 48 & g__Turicibacter & 27 & 0.01 \\
\hline 49 & g__Aeromicrobium & 15 & 0.01 \\
\hline 50 & g_Blastococcus & 15 & 0.01 \\
\hline
\end{tabular}

Table S5f: Core Bacteriome: The core Bacteriome analysis was performed on the set of 224 OTUs filtered through the MicrobiomeAnalyst. The below taxa with a sample prevalence of $20 \%$ and a relative abundance above 0.01 was obtained as the core bacteriome.

\begin{tabular}{|l|r|}
\hline & Prevelance \\
\hline g__Brevundimonas & 1 \\
\hline g__Mesorhizobium & 1 \\
\hline Not_Assigned & 1 \\
\hline g__Allorhizobium & 0.8 \\
\hline g___assilia & 0.8 \\
\hline g__Devosia & 0.75 \\
\hline g__Pseudomonas & 0.75 \\
\hline g_Achromobacter & 0.65 \\
\hline g__Sphingopyxis & 0.65 \\
\hline g_Azotobacter & 0.45 \\
\hline g__Microvirga & 0.4 \\
\hline g_Sphingomonas & 0.4 \\
\hline g__Stenotrophomonas & 0.35 \\
\hline g_Acidovorax & 0.3 \\
\hline g__Cavicella & 0.3 \\
\hline g__Novosphingobium & 0.3 \\
\hline g_Parvibaculum & 0.3 \\
\hline g_Pseudoxanthomonas & 0.3 \\
\hline g_uncultured & 0.3 \\
\hline g__Variovorax & 0.3 \\
\hline g__Bacillus & 0.25 \\
\hline g__Halomonas & 0.25 \\
\hline g__Hydrocarboniphaga & 0.25 \\
\hline g__Pseudarthrobacter & 0.25 \\
\hline
\end{tabular}




\begin{tabular}{|l|r|}
\hline g__Sphingobium & 0.25 \\
\hline g__Corynebacterium_1 & 0.2 \\
\hline g__Pontibacter & 0.2 \\
\hline g__Rhizobacter & 0.2 \\
\hline g__Shewanella & 0.2 \\
\hline g__Bradyrhizobium & 0.15 \\
\hline g__Enhydrobacter & 0.15 \\
\hline g__Cutibacterium & 0.1 \\
\hline g__Delftia & 0.1 \\
\hline g__Hahella & 0.05 \\
\hline g__Kocuria & 0.05 \\
\hline g__Methylobacterium & 0.05 \\
\hline g__Micrococcus & 0.05 \\
\hline g__Noviherbaspirillum & 0.05 \\
\hline g__Paracoccus & 0.05 \\
\hline g__Ramlibacter & 0.05 \\
\hline g__Salinimicrobium & 0.05 \\
\hline g__Staphylococcus & 0.05 \\
\hline g__Tetrasphaera & 0.05 \\
\hline g__Aeromicrobium & 0 \\
\hline g__Blastococcus & 0 \\
\hline g__Brachybacterium & 0 \\
\hline g__Clostridium_sensu_stricto_1 & 0 \\
\hline g__Romboutsia & 0 \\
\hline g__Skermanella & 0 \\
\hline g__Turicibacter & 0 \\
\hline &
\end{tabular}




\section{Fungal Taxonomy}

Table S5g: Fungal Taxonomic classification at Phylum level

\begin{tabular}{|r|l|r|r|}
\hline & Taxa & Abundance & Percentage \\
\hline 1 & p_Ascomycota & 347907 & 64.19 \\
\hline 2 & p__Basidiomycota & 122714 & 22.64 \\
\hline 3 & Unclassified & 64018 & 11.81 \\
\hline 4 & p_Zygomycota & 7321 & 1.35 \\
\hline
\end{tabular}

Table S5h: Fungal Taxonomic Classification at Class level

\begin{tabular}{|r|l|r|r|}
\hline & Taxa & Abundance & Percentage \\
\hline 3 & c__Dothideomycetes & 190556 & 35.16 \\
\hline 4 & Not_Assigned & 95622 & 17.64 \\
\hline 8 & c__Tremellomycetes & 68808 & 12.7 \\
\hline 5 & c__Eurotiomycetes & 52968 & 9.77 \\
\hline 1 & c__Sordariomycetes & 36534 & 6.74 \\
\hline 7 & c__Agaricomycetes & 33707 & 6.22 \\
\hline 10 & c__Saccharomycetes & 25077 & 4.63 \\
\hline 2 & c__ncertae_sedis & 24110 & 4.45 \\
\hline 9 & c_Leotiomycetes & 8393 & 1.55 \\
\hline 11 & c__Microbotryomycetes & 2775 & 0.51 \\
\hline 6 & c_Pezizomycetes & 2166 & 0.4 \\
\hline 14 & c__unidentified & 882 & 0.16 \\
\hline 12 & c_Wallemiomycetes & 203 & 0.04 \\
\hline 13 & c__Ustilaginomycetes & 83 & 0.02 \\
\hline 15 & c__Exobasidiomycetes & 76 & 0.01 \\
\hline
\end{tabular}

Table S5i: Fungal Taxonomic Classification at Order level

\begin{tabular}{|r|l|r|r|}
\hline & Taxa & Abundance & Percentage \\
\hline 13 & o__Capnodiales & 104331 & 19.25 \\
\hline 4 & Not_Assigned & 97407 & 17.97 \\
\hline 3 & o__Pleosporales & 83150 & 15.34 \\
\hline 9 & o__Filobasidiales & 66286 & 12.23 \\
\hline 5 & o__Eurotiales & 48019 & 8.86 \\
\hline 12 & o__Hypocreales & 29173 & 5.38 \\
\hline 11 & o__Saccharomycetales & 25085 & 4.63 \\
\hline 7 & o__Polyporales & 23711 & 4.38 \\
\hline 2 & o__Malasseziales & 16170 & 2.98 \\
\hline 20 & o__Agaricales & 8894 & 1.64 \\
\hline
\end{tabular}




\begin{tabular}{|r|l|r|r|}
\hline 10 & o__Helotiales & 7531 & 1.39 \\
\hline 24 & o__Mucorales & 3979 & 0.73 \\
\hline 1 & o__Microascales & 3817 & 0.7 \\
\hline 17 & o__Chaetothyriales & 3380 & 0.62 \\
\hline 22 & o__Mortierellales & 3329 & 0.61 \\
\hline 27 & o__Dothideales & 3159 & 0.58 \\
\hline 15 & o__Sporidiobolales & 2784 & 0.51 \\
\hline 16 & o__Sordariales & 2536 & 0.47 \\
\hline 23 & o__Tremellales & 2116 & 0.39 \\
\hline 6 & o__ezizales & 1918 & 0.35 \\
\hline 19 & o__Onygenales & 1244 & 0.23 \\
\hline 26 & o__unidentified & 1147 & 0.21 \\
\hline 21 & o__Erysiphales & 911 & 0.17 \\
\hline 14 & o__Hymenochaetales & 818 & 0.15 \\
\hline 25 & o__Incertae_sedis & 699 & 0.13 \\
\hline 18 & o__Wallemiales & 204 & 0.04 \\
\hline 28 & o__Ustilaginales & 87 & 0.02 \\
\hline 8 & o__Xylariales & 75 & 0.01 \\
\hline
\end{tabular}

Table S5j: Fungal Taxonomic Classification at Family level

\begin{tabular}{|r|l|r|r|}
\hline & Taxa & Abundance & Percentage \\
\hline 2 & Not_Assigned & 149342 & 27.56 \\
\hline 8 & f__Mycosphaerellaceae & 97185 & 17.93 \\
\hline 6 & f__Filobasidiaceae & 66239 & 12.22 \\
\hline 3 & f_Pleosporaceae & 62007 & 11.44 \\
\hline 4 & f__Trichocomaceae & 48028 & 8.86 \\
\hline 7 & f_Incertae_sedis & 36588 & 6.75 \\
\hline 10 & f_unidentified & 18877 & 3.48 \\
\hline 21 & f_Nectriaceae & 8997 & 1.66 \\
\hline 35 & f__Schizophyllaceae & 8622 & 1.59 \\
\hline 19 & f_Sporormiaceae & 5822 & 1.07 \\
\hline 27 & f__Mucoraceae & 3993 & 0.74 \\
\hline 1 & f_Microascaceae & 3766 & 0.69 \\
\hline 12 & f__Herpotrichiellaceae & 3386 & 0.62 \\
\hline 24 & f__Mortierellaceae & 3333 & 0.61 \\
\hline 31 & f__Dothioraceae & 2903 & 0.54 \\
\hline 15 & f_Clavicipitaceae & 2395 & 0.44 \\
\hline 38 & f_Teratosphaeriaceae & 2286 & 0.42 \\
\hline 25 & f_Trichosporonaceae & 2120 & 0.39 \\
\hline 37 & f__Hypocreaceae & 2000 & 0.37 \\
\hline
\end{tabular}




\begin{tabular}{|r|l|r|r|}
\hline 28 & f__Polyporaceae & 1915 & 0.35 \\
\hline 11 & f__Chaetomiaceae & 1716 & 0.32 \\
\hline 5 & f__Pezizaceae & 1530 & 0.28 \\
\hline 33 & f__Tubeufiaceae & 1268 & 0.23 \\
\hline 16 & f__Onygenaceae & 1087 & 0.2 \\
\hline 18 & f__Fomitopsidaceae & 1062 & 0.2 \\
\hline 23 & f__Erysiphaceae & 908 & 0.17 \\
\hline 9 & f__Hymenochaetaceae & 828 & 0.15 \\
\hline 13 & f__Cordycipitaceae & 777 & 0.14 \\
\hline 26 & f__Lasiosphaeriaceae & 734 & 0.14 \\
\hline 22 & f__Debaryomycetaceae & 707 & 0.13 \\
\hline 29 & f__Sclerotiniaceae & 591 & 0.11 \\
\hline 34 & f__Ascobolaceae & 212 & 0.04 \\
\hline 14 & f__Wallemiaceae & 210 & 0.04 \\
\hline 17 & f__Gymnoascaceae & 209 & 0.04 \\
\hline 20 & f__Marasmiaceae & 130 & 0.02 \\
\hline 32 & f__Ustilaginaceae & 87 & 0.02 \\
\hline 36 & f__Saccharomycodaceae & 54 & 0.01 \\
\hline 30 & f__Psathyrellaceae & 46 & 0.01 \\
\hline
\end{tabular}

Table S5k: Fungal Taxonomic Classification at Genus level

\begin{tabular}{|r|l|r|r|}
\hline & Taxa & Abundance & Percentage \\
\hline 2 & Not_Assigned & 172419 & 31.81 \\
\hline 5 & g__unidentified & 102488 & 18.91 \\
\hline 9 & g__Cryptococcus & 65663 & 12.12 \\
\hline 3 & g__Alternaria & 46554 & 8.59 \\
\hline 7 & g__Aspergillus & 36298 & 6.7 \\
\hline 11 & g__Candida & 16577 & 3.06 \\
\hline 12 & g__Cladosporium & 15920 & 2.94 \\
\hline 56 & g__Schizophyllum & 8622 & 1.59 \\
\hline 31 & g__Fusarium & 7511 & 1.39 \\
\hline 10 & g__Gloeotinia & 6920 & 1.28 \\
\hline 4 & g__Penicillium & 6216 & 1.15 \\
\hline 18 & g__Pleospora & 4189 & 0.77 \\
\hline 42 & g__Mucor & 3993 & 0.74 \\
\hline 37 & g__Mortierella & 3333 & 0.61 \\
\hline 30 & g__Eurotium & 3010 & 0.56 \\
\hline 52 & g__Aureobasidium & 2903 & 0.54 \\
\hline 8 & g__Cochliobolus & 2661 & 0.49 \\
\hline 23 & g__Malassezia & 2609 & 0.48 \\
\hline
\end{tabular}




\begin{tabular}{|c|c|c|c|}
\hline 24 & g__Rhodotorula & 2299 & 0.42 \\
\hline 63 & g__Hortaea & 2286 & 0.42 \\
\hline 40 & g_trichosporon & 2120 & 0.39 \\
\hline 46 & g_Exophiala & 1944 & 0.36 \\
\hline 38 & g_Beauveria & 1609 & 0.3 \\
\hline 55 & g__Gibberella & 1486 & 0.27 \\
\hline 45 & g_Cerrena & 1435 & 0.26 \\
\hline 39 & g__Scedosporium & 1420 & 0.26 \\
\hline 49 & g__Chaetomium & 1369 & 0.25 \\
\hline 54 & $\mathrm{~g}$ _ Thaxteriellopsis & 1268 & 0.23 \\
\hline 28 & g__Setosphaeria & 1191 & 0.22 \\
\hline 61 & g__Cercospora & 1077 & 0.2 \\
\hline 27 & g__Fomitopsis & 1062 & 0.2 \\
\hline 60 & g_Trichoderma & 999 & 0.18 \\
\hline 13 & g__Phellinus & 828 & 0.15 \\
\hline 20 & g_Lecanicillium & 777 & 0.14 \\
\hline 6 & g_Emericella & 739 & 0.14 \\
\hline 35 & $\mathrm{~g}$ _Blumeria & 731 & 0.13 \\
\hline 34 & g__Meyerozyma & 707 & 0.13 \\
\hline 41 & g__Doratomyces & 668 & 0.12 \\
\hline 22 & g__Myrothecium & 643 & 0.12 \\
\hline 44 & g__Tritirachium & 602 & 0.11 \\
\hline 47 & g_Botryotinia & 591 & 0.11 \\
\hline 33 & $\mathrm{~g}$ _Cordyceps & 579 & 0.11 \\
\hline 19 & g_Kodamaea & 570 & 0.11 \\
\hline 51 & $\mathrm{~g} \_$Acremonium & 560 & 0.1 \\
\hline 62 & g__Megasporoporia & 480 & 0.09 \\
\hline 14 & g__Sporobolomyces & 453 & 0.08 \\
\hline 17 & g_Fonsecaea & 438 & 0.08 \\
\hline 48 & g_Cladorrhinum & 363 & 0.07 \\
\hline 1 & g__Microascus & 302 & 0.06 \\
\hline 36 & g__Bipolaris & 290 & 0.05 \\
\hline 15 & g_Pseudallescheria & 276 & 0.05 \\
\hline 32 & g_Lewia & 263 & 0.05 \\
\hline 16 & g__Stachybotrys & 213 & 0.04 \\
\hline 21 & $\mathrm{~g}$ _Wallemia & 210 & 0.04 \\
\hline 26 & g__Gymnascella & 209 & 0.04 \\
\hline 58 & g__Meira & 183 & 0.03 \\
\hline 64 & g__Erysiphe & 177 & 0.03 \\
\hline 43 & g_Pyrenophora & 161 & 0.03 \\
\hline 25 & g__Metarhizium & 130 & 0.02 \\
\hline
\end{tabular}




\begin{tabular}{|r|l|r|r|}
\hline 29 & g__Marasmius & 130 & 0.02 \\
\hline 53 & g__Ustilago & 87 & 0.02 \\
\hline 57 & g__Hanseniaspora & 54 & 0.01 \\
\hline 59 & g__Scopulariopsis & 49 & 0.01 \\
\hline 50 & g__Coprinellus & 46 & 0.01 \\
\hline
\end{tabular}

Table S51: Fungal Taxonomic classification at species level

\begin{tabular}{|c|c|c|c|}
\hline & Taxa & Abundance & Percentage \\
\hline 1 & Not_Assigned & 225762 & 41.66 \\
\hline 2 & S__unculturedCladosporium & 80188 & 14.8 \\
\hline 3 & s_Alternaria_brassicae & 46447 & 8.57 \\
\hline 4 & S_Cryptococcus_oeirensis & 30610 & 5.65 \\
\hline 5 & s_Cryptococcus_albidus & 21755 & 4.01 \\
\hline 6 & s_Candida_parapsilosis & 13002 & 2.4 \\
\hline 7 & s_Aspergillus_gracilis & 10667 & 1.97 \\
\hline 8 & s__Aspergillus_penicillioides & 9536 & 1.76 \\
\hline 9 & s__unculturedfungus & 9376 & 1.73 \\
\hline 10 & s__Schizophyllum_commune & 8622 & 1.59 \\
\hline 11 & s_Gloeotinia_temulenta & 6920 & 1.28 \\
\hline 12 & s_Cryptococcus_carnescens & 5205 & 0.96 \\
\hline 13 & s_Capnodiales_sp_TR006 & 4385 & 0.81 \\
\hline 14 & s_Aspergillus_versicolor & 3060 & 0.56 \\
\hline 15 & s_Cryptococcus_stepposus & 2738 & 0.51 \\
\hline 16 & s__Aureobasidium_pullulans & 2702 & 0.5 \\
\hline 17 & s__unculturedMalassezia & 2584 & 0.48 \\
\hline 18 & s_Cryptococcus_laurentii & 2390 & 0.44 \\
\hline 19 & s__Malassezia_globosa & 2334 & 0.43 \\
\hline 20 & s__Rhodotorula_mucilaginosa & 2299 & 0.42 \\
\hline 21 & s__Hortaea_werneckii & 2286 & 0.42 \\
\hline 22 & s__Trichosporon_asahii & 2120 & 0.39 \\
\hline 23 & s_Cryptococcus_magnus & 2083 & 0.38 \\
\hline 24 & s_Exophiala_dermatitidis & 1944 & 0.36 \\
\hline 25 & s_Pleospora_herbarum & 1846 & 0.34 \\
\hline 26 & s_Candida_sp_F15 & 1812 & 0.33 \\
\hline 27 & s__unculturedEurotium & 1765 & 0.33 \\
\hline 28 & s_Aspergillus_niger & 1732 & 0.32 \\
\hline 29 & s_Candida_tropicalis & 1639 & 0.3 \\
\hline 30 & s_Beauveria_bassiana & 1609 & 0.3 \\
\hline 31 & s__Fusarium_oxysporum & 1594 & 0.29 \\
\hline 33 & s__Eurotium_niveoglaucum & 1542 & 0.28 \\
\hline
\end{tabular}




\begin{tabular}{|c|c|c|c|}
\hline 32 & s_Pezizaceae_sp_F13 & 1530 & 0.28 \\
\hline 35 & s__Cerrena_unicolor & 1435 & 0.26 \\
\hline 34 & s__Scedosporium_prolificans & 1420 & 0.26 \\
\hline 36 & s_CChaetomium_globosum & 1369 & 0.25 \\
\hline 37 & s_Aspergillus_peyronelii & 1270 & 0.23 \\
\hline 38 & s__Thaxteriellopsis_lignicola & 1268 & 0.23 \\
\hline 39 & S_Cochliobolus_sp_FSU9312 & 1210 & 0.22 \\
\hline 40 & S__Setosphaeria_rostrata & 1191 & 0.22 \\
\hline 42 & s_Cercospora_capsici & 1077 & 0.2 \\
\hline 41 & s_FFomitopsis_sp_CLF_T & 1062 & 0.2 \\
\hline 43 & s_Gibberella_intermedia & 977 & 0.18 \\
\hline 44 & s_Cochliobolus_sp_007 L 1_1 & 896 & 0.17 \\
\hline 45 & S_Phellinus_noxius & 828 & 0.15 \\
\hline 47 & s_Cryptococcus_dimennae & 777 & 0.14 \\
\hline 48 & s__Lecanicillium_fusisporum & 777 & 0.14 \\
\hline 46 & s_Emericella_nidulans & 739 & 0.14 \\
\hline 49 & S_Blumeria_graminis & 731 & 0.13 \\
\hline 53 & s_Tritirachium_sp_IAM_14522 & 602 & 0.11 \\
\hline 52 & s__Cladosporium_sp_B09 & 591 & 0.11 \\
\hline 54 & s_Botryotinia_fuckeliana & 591 & 0.11 \\
\hline 51 & S_Penicillium_capsulatum & 573 & 0.11 \\
\hline 50 & s_Kodamaea_ohmeri & 570 & 0.11 \\
\hline 56 & S_CCochliobolus_sativus & 555 & 0.1 \\
\hline 55 & s_Cordyceps_chlamydosporia & 554 & 0.1 \\
\hline 57 & s__Gibberella_zeae & 509 & 0.09 \\
\hline 58 & s__Megasporoporia_sp_HJL_2012d & 480 & 0.09 \\
\hline 59 & s__Fonsecaea_pedrosoi & 438 & 0.08 \\
\hline 60 & s_Myrothecium_verrucaria & 435 & 0.08 \\
\hline 61 & s__Cladorrhinum_bulbillosum & 363 & 0.07 \\
\hline 64 & s_Cladosporium_sp_ZJ_2008016 & 342 & 0.06 \\
\hline 63 & s__Doratomyces_sp_HZ_10 & 322 & 0.06 \\
\hline 62 & s__Microascus_sp_4L1 & 302 & 0.06 \\
\hline 65 & S_Pseudallescheria_fimeti & 276 & 0.05 \\
\hline 67 & S__Malassezia_sympodialis & 275 & 0.05 \\
\hline 66 & s_Pezizomycetes_sp_BLD9 & 252 & 0.05 \\
\hline 68 & S_Stachybotrys_chlorohalonata & 213 & 0.04 \\
\hline 70 & s__Gymnascella_aurantiaca & 209 & 0.04 \\
\hline 69 & S__Myrothecium_gramineum & 208 & 0.04 \\
\hline 72 & s_Aspergillus_terreus & 185 & 0.03 \\
\hline 75 & s__Meira_sp_VegaE2_32 & 183 & 0.03 \\
\hline 76 & s_Erysiphe_convolvuli & 177 & 0.03 \\
\hline
\end{tabular}




\begin{tabular}{|r|l|r|r|}
\hline 71 & s_Penicillium_chrysogenum & 166 & 0.03 \\
\hline 74 & s_Pyrenophora_graminea & 161 & 0.03 \\
\hline 73 & s__Pezizales_sp_P10 & 149 & 0.03 \\
\hline 79 & s__Metarhizium_anisopliae & 130 & 0.02 \\
\hline 80 & s__Marasmius_leveilleanus & 130 & 0.02 \\
\hline 78 & s_unculturedThielavia & 128 & 0.02 \\
\hline 83 & s__Sporobolomyces_roseus & 126 & 0.02 \\
\hline 82 & s_Candida_orthopsilosis & 124 & 0.02 \\
\hline 77 & s_Cryptococcus_sp_HB_1222 & 105 & 0.02 \\
\hline 81 & s__Ustilago_hordei & 87 & 0.02 \\
\hline 86 & s_Acremonium_implicatum & 80 & 0.01 \\
\hline 84 & s_Hypocreales_sp_SCSGAF0072 & 77 & 0.01 \\
\hline 88 & s_Cladosporium_sp_F128 & 62 & 0.01 \\
\hline 87 & s_Hanseniaspora_uvarum & 54 & 0.01 \\
\hline 85 & s_Coprinellus_sp_Daams_72_68a & 46 & 0.01 \\
\hline 89 & s_Aspergillus_caespitosus & 19 & 0 \\
\hline
\end{tabular}

Table S5m Core Fungal Biome: The core fungal biome analysis was performed on the set of 371 OTUs filtered through the MicrobiomeAnalyst. A set of 65 taxa with a sample prevalence of $20 \%$ and a relative abundance above 0.01 was obtained as the core microbiome.

\begin{tabular}{|l|r|r|}
\hline & Prevelance \\
\hline Not_Assigned & 1 & 100 \\
\hline g__unidentified & 1 & 100 \\
\hline g__Cryptococcus & 1 & 100 \\
\hline g__Alternaria & 1 & 100 \\
\hline g__Aspergillus & 0.9 & 90 \\
\hline g__Cladosporium & 0.75 & 75 \\
\hline g__Fusarium & 0.6 & 60 \\
\hline g__Candida & 0.55 & 55 \\
\hline g__Schizophyllum & 0.3 & 30 \\
\hline g__Penicillium & 0.25 & 25 \\
\hline g__Pleospora & 0.2 & 20 \\
\hline g__Mucor & 0.2 & 20 \\
\hline g__Cochliobolus & 0.2 & 20 \\
\hline g__Aureobasidium & 0.2 & 20 \\
\hline g__Scedosporium & 0.15 & 15 \\
\hline g__Mortierella & 0.15 & 15 \\
\hline g__Malassezia & 0.15 & 15 \\
\hline g__Hortaea & 0.15 & 15 \\
\hline g__Thaxteriellopsis & 0.1 & 10 \\
\hline
\end{tabular}




\begin{tabular}{|c|c|c|}
\hline g__Gloeotinia & 0.1 & 10 \\
\hline g__Eurotium & 0.1 & 10 \\
\hline g__Chaetomium & 0.1 & 10 \\
\hline g__Cerrena & 0.1 & 10 \\
\hline g__ Tritirachium & 0.05 & 5 \\
\hline g__Trichosporon & 0.05 & 5 \\
\hline g__Setosphaeria & 0.05 & 5 \\
\hline g__ Rhodotorula & 0.05 & 5 \\
\hline g__Phellinus & 0.05 & 5 \\
\hline g__Lecanicillium & 0.05 & 5 \\
\hline g__Gibberella & 0.05 & 5 \\
\hline g__Fonsecaea & 0.05 & 5 \\
\hline $\mathrm{g} \_$Fomitopsis & 0.05 & 5 \\
\hline g_Exophiala & 0.05 & 5 \\
\hline g__Emericella & 0.05 & 5 \\
\hline g__Cordyceps & 0.05 & 5 \\
\hline g__Cercospora & 0.05 & 5 \\
\hline g__Blumeria & 0.05 & 5 \\
\hline g__Beauveria & 0.05 & 5 \\
\hline g__Wallemia & 0 & 0 \\
\hline g__Ustilago & 0 & 0 \\
\hline g__Trichoderma & 0 & 0 \\
\hline g__Stachybotrys & 0 & 0 \\
\hline g__Sporobolomyces & 0 & 0 \\
\hline g__Scopulariopsis & 0 & 0 \\
\hline g__Pyrenophora & 0 & 0 \\
\hline g__Pseudallescheria & 0 & 0 \\
\hline g__Myrothecium & 0 & 0 \\
\hline g___Microascus & 0 & 0 \\
\hline g__Meyerozyma & 0 & 0 \\
\hline g__Meira & 0 & 0 \\
\hline g__Megasporoporia & 0 & 0 \\
\hline g__ Marasmius & 0 & 0 \\
\hline g__Lewia & 0 & 0 \\
\hline g__Kodamaea & 0 & 0 \\
\hline g__Hanseniaspora & 0 & 0 \\
\hline g__Gymnascella & 0 & 0 \\
\hline g__Erysiphe & 0 & 0 \\
\hline g__Doratomyces & 0 & 0 \\
\hline g_Coprinellus & 0 & 0 \\
\hline g__Cladorrhinum & 0 & 0 \\
\hline
\end{tabular}




\begin{tabular}{|l|l|l|}
\hline g__Botryotinia & 0 & 0 \\
\hline g__Bipolaris & 0 & 0 \\
\hline g_Acremonium & 0 & 0 \\
\hline
\end{tabular}

